\title{
Directional Control of Mature Osteoblast Derived from Juvenile Mouse Calvariae
}

\author{
Ryosuke Ozasa $^{1, * 1}$, Aira Matsugaki ${ }^{1}$, Yoshihiro Isobe ${ }^{2}$, Taro Saku ${ }^{2}$ and Takayoshi Nakano ${ }^{1, * 2}$ \\ ${ }^{1}$ Division of Materials Science and Engineering, Graduate School of Engineering, Osaka University, Suita 565-0871, Japan \\ ${ }^{2}$ Atree, Inc, Tokyo 150-0012, Japan
}

Bone tissue has a highly anisotropic microstructure comprised of biological apatite (BAp) and collagen fibrils, which is closely related to bone mechanical function. The formation of anisotropic bone microstructure is governed by bone-forming osteoblasts; therefore, isolation of functional osteoblasts and regulation of their arrangement for generating bone tissue with optimally-oriented microstructure during bone reconstruction are important. In this study, we established the isolation and culture conditions of mature osteoblasts derived from juvenile mice (2-week-old). Osteoblasts from juvenile mice expressed significantly higher level of osteoblastic markers (alkaline phosphatase, osterix, and osteocalcin) than osteoblasts from neonatal mice, indicating that juvenile osteoblasts are promising materials for bone tissue engineering. Moreover, alignment of the mature osteoblasts was successfully regulated using oriented collagen substrates. This is the report that shows directional control of mature osteoblasts isolated from juvenile mice. [doi:10.2320/matertrans.M2017077]

(Received March 9, 2017; Accepted March 29, 2017; Published April 28, 2017)

Keywords: bone anisotropy, primary osteoblast, isolation method, mature cell, cell orientation, oriented collagen substrate

\section{Introduction}

The morphology of a living tissue or organ is closely associated with its functions. In particular, bone tissue has a highly anisotropic microstructure with parallel arrangement of biological apatite (BAp)/collagen composites along the long bone axis, ${ }^{1)}$ which governs its mechanical function. ${ }^{2-4)}$ During bone formation, anisotropic bone microstructure is constructed by osteoblasts, the bone-forming cells adhering to the bone surface. We have reported that the degree of bone matrix anisotropy correlates quantitatively to the level of osteoblast alignment. ${ }^{5)}$ Therefore, isolation of functional osteoblasts and regulation of their arrangement for realizing ideal bone tissue constructs with optimally oriented microstructure is important.

Currently, osteoblasts derived from neonatal mice have been used as the source of primary osteoblasts because of the simplicity of the cell culture procedure. ${ }^{6)}$ However, certain features of osteoblasts derived from neonatal mice (neonatal osteoblast) are inferior compared to those of the mature osteoblasts. For instance, neonatal osteoblasts are relatively immature with lower mineralization activity. ${ }^{7)}$ Therefore, optimization of isolation and culture of mature osteoblasts, and directional regulation of the obtained osteoblasts are necessary for establishing bone matrix anisotropy by controlling cellular behaviors.

In this study, we optimized the isolation and subsequent culturing of homogeneous mature osteoblasts. In addition, the preferential orientation of the osteoblasts was regulated using oriented collagen substrates as cell scaffolds, ${ }^{8)}$ which is crucial for constructing the anisotropic bone microstructure. ${ }^{5)}$ Our results clearly highlight the potential of juvenile mouse-derived osteoblasts (juvenile osteoblast) in construction of bone tissue with the original anisotropic microstructure.

\footnotetext{
${ }^{*}$ Graduate Student, Osaka University

*2 Corresponding author, E-mail: nakano@mat.eng.osaka-u.ac.jp
}

\section{Materials and Methods}

\subsection{Osteoblast isolation from juvenile mice and cell cul- ture}

Primary osteoblast was isolated based on the method of primary osteoblast isolation from adult mice ${ }^{9)}$ with certain modification as mentioned below. Juvenile (2-week-old) or adult (8-week-old) C57BL6 mice were sacrificed by cervical dislocation, and the skin was removed from the skulls. Then, calvariae were excised and plated in ice-cold $\alpha$-minimum essential medium ( $\alpha$-MEM; GIBCO, Invitrogen, CA, USA). For isolation of the homogeneous osteoblast population, limited areas of the forehead and parietal bone (areas surrounded by dashed line in Fig. 1) were carefully dissected from a special part of the calvariae, and the fibrous tissue around the bone was removed under the microscope. The isolated bone was cut into 1-2 mm small fragments and washed with Hanks balanced salt solution (HBSS; GIBCO) to remove the $\alpha$-MEM. Subsequently, the bone fragments were exposed to a series of enzymatic digestion. At the $1^{\text {st }}, 2^{\text {nd }}$ and final digestion, the bone fragments were incubated in collagenase solution $(50 \mu \mathrm{L} 2.5 \mathrm{w} / \mathrm{v} \%$ collagenase I; WAKO, Osaka, Japan, in $\mathrm{HBSS}$ and $450 \mu \mathrm{L} \mathrm{HBSS}$ ) at $37^{\circ} \mathrm{C}$ for $30 \mathrm{~min}$. At the $3^{\text {rd }} \mathrm{di}-$ gestion, the bone fragments were incubated with trypsin solution $(500 \mu \mathrm{L} 0.25 \mathrm{w} / \mathrm{v} \%$ trypsin/EDTA; Nacalai Tesque, Kyoto, Japan) at $37^{\circ} \mathrm{C}$ for $30 \mathrm{~min}$. Then, $\alpha$-MEM containing $10 \%$ fetal bovine serum (FBS; GIBCO) was added three times totally to inhibit the enzymatic activity. The digested bone fragments were explant-cultured in $\alpha$-MEM containing $10 \% \mathrm{FBS}$ for 2 weeks at $37^{\circ} \mathrm{C}$ in $5 \% \mathrm{CO}_{2}$. The culture medium was changed thrice a week. The cultured cells were detached by trypsinization, and passage 1 (P1) osteoblasts from juvenile mice were used in experiments. (Fig. 1)

\subsection{Osteoblast isolation from neonatal mice and cell cul- ture}

Neonatal osteoblast isolation was performed using the sequential digestion protocol. ${ }^{6)}$ Neonatal C57BL/6 mice were sacrificed by decapitation, following which the calvariae were excised and placed in ice-cold $\alpha$-MEM, and the fibrous 


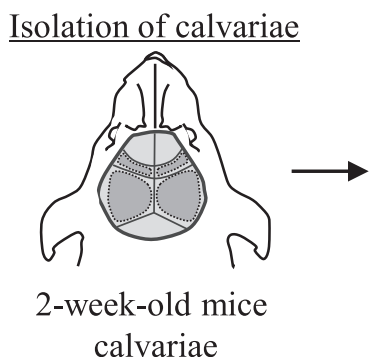

calvariae
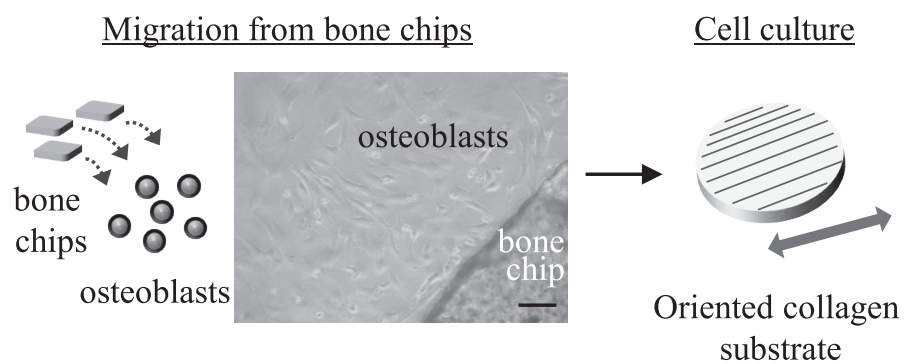

Fig. 1 Schematic illustration of the procedure of primary osteoblast culture on oriented collagen substrate. Phase contrast image indicates osteoblasts migrating from bone chip. Scale bars $=100 \mu \mathrm{m}$.

tissues around the bone were gently removed under a microscope. The outer layers of the periosteum were removed to isolate a highly homogeneous osteoblast population. Then, the calvariae were subjected to a series of collagenase/trypsin digestions at $37^{\circ} \mathrm{C}$ for $15 \mathrm{~min}$ each. The $3^{\text {rd }}, 4^{\text {th }}$, and $5^{\text {th }}$ supernatants were collected in $\alpha$-MEM to inhibit the enzymatic activity, pooled, and filtered using a $200-\mu \mathrm{m}$ mesh. The cells were centrifuged and the supernatants were discarded. Remaining cell pellets were resuspended in $\alpha$-MEM containing $10 \%$ FBS. The cells were cultured in $\alpha$-MEM containing $10 \% \mathrm{FBS}$ at $37^{\circ} \mathrm{C}$ in $5 \% \mathrm{CO}_{2}$. The culture medium was changed twice a week.

\subsection{Gene expression analysis}

The total RNA was extracted by using Trizol (Invitrogen) according to the manufacturer's instructions, and quantified using a biophotometer (Eppendorf, Hamburg, Germany). mRNA $(0.3 \mu \mathrm{g})$ was used to analyze the expression of the osteoblastic differentiation markers, namely, $C O L-I$ (collagen type I), ALP (alkaline phosphatase), OSX (osterix), and $B G L A P$ (osteocalcin), using the StepOne real time polymerase chain reaction (PCR) system (Applied Biosystems, Invitrogen). The values obtained for each marker was normalized to that of GAPDH $(\mathrm{n}=3)$.

\subsection{Control of cell alignment}

The juvenile osteoblasts were cultured for 3 days on oriented collagen substrates prepared by a hydrodynamic extrusion method. ${ }^{8)}$ Osteoblasts were fixed in $4 \%$ formaldehyde/phosphate-buffered saline (PBS) for 20 min followed by washing thrice with PBS/Triton-X (PBST). The cells were blocked using $1 \%$ normal goat serum (NGS; Invitrogen) in PBST for $30 \mathrm{~min}$. Then, the cells were incubated with primary antibodies (anti Src; Cell Signaling, MA, USA, or anti vinculin; Sigma, MO, USA) at $4^{\circ} \mathrm{C}$ for $12 \mathrm{~h}$, followed by washing in PBST, and further incubated with secondary antibodies at room temperature for 90 min and Alexa Fluor ${ }^{\circledR} 488$-conjugated phalloidin (Invitrogen) at room temperature for $20 \mathrm{~min}$. Thereafter, the cells were washed by PBST and mounted in Prolong Gold antifade reagent with DAPI (Invitrogen). Phase contrast and fluorescent images were obtained using an optical microscope (Biozero, Keyence, Osaka, Japan). The images were analyzed with Image $\mathbf{J}$ software (NIH, MD, USA) $(n=3$ in each group). Cell orientation was quantitatively analyzed using the Cell Profiler software (Broad Institute, Cambridge, MA). The degree of cell orientation was characterized by the angular standard deviation, S.D. (s), for a wrapped normal distribution. ${ }^{10,11)}$ Here, the probability distribution function was adapted from Fisher ${ }^{12)}$ for a periodicity of $\pi$ radians, where $\mu$ is the mean angle and $\rho$ is the mean resultant length. These parameters were determined from a set of $n$ measured cell orientations, $\theta_{i}$, by the following equations:

$$
\begin{gathered}
f(\theta)=1 / \pi\left(1+2 \sum_{p=1}^{\infty} \rho^{p^{2}} \cos (2 p(\theta-\pi))\right) \\
p=1 / n \sqrt{\left(\sum_{i=1}^{n} \cos 2 \theta_{i}\right)^{2}+\left(\sum_{i=1}^{n} \sin 2 \theta_{i}\right)^{2}} \\
\mu=\tan ^{-1}\left(\sum_{i=1}^{n} \sin 2 \theta_{i} / \sum_{i=1}^{n} \cos 2 \theta_{i}\right) .
\end{gathered}
$$

The angular standard deviation $(\sigma)$ was determined using the following equation:

$$
\sigma=1 / 2 \sqrt{-2 \ln \rho}
$$

\subsection{Statistical analysis}

Statistical significance was determined using the Student's $t$-test. $P<0.01$ was considered significant.

\section{Results and Discussion}

\subsection{Isolation of primary osteoblasts from juvenile mice}

Following enzymatic digestion, the juvenile osteoblasts migrated from the bone fragments (Fig. 1) and reached confluence after 2 weeks of explant-culture. In contrast to the osteoblasts isolated from adult mice (adult osteoblasts) (Fig. 2A), the isolated juvenile osteoblasts (Fig. 2B) were not contaminated with other cell types such as lymphoid cells and myeloid cells, which generally increase with aging in the calvariae, ${ }^{13)}$ suggesting that age of the donor mice, origin, and the isolation method used in this study were suitable for obtaining homogeneous osteoblast population. The isolated juvenile osteoblasts were round and contained actin stress fibers and focal adhesions positive for c-src, which mediate several essential biological signaling cascades $^{14)}$; this confirmed that the juvenile osteoblasts possessed high cell activity because the formation of actin stress fibers strongly correlates with the dynamic cell behavior such as cell migration. ${ }^{15)}$ Thus, a homogeneous osteoblast population was successfully obtained from the calvariae of juvenile mice.

Osteoblasts isolated from tissues show different properties depending on their origin or the age of the donor animals. ${ }^{7)}$ Immature osteoblasts from neonatal mice have higher prolif- 

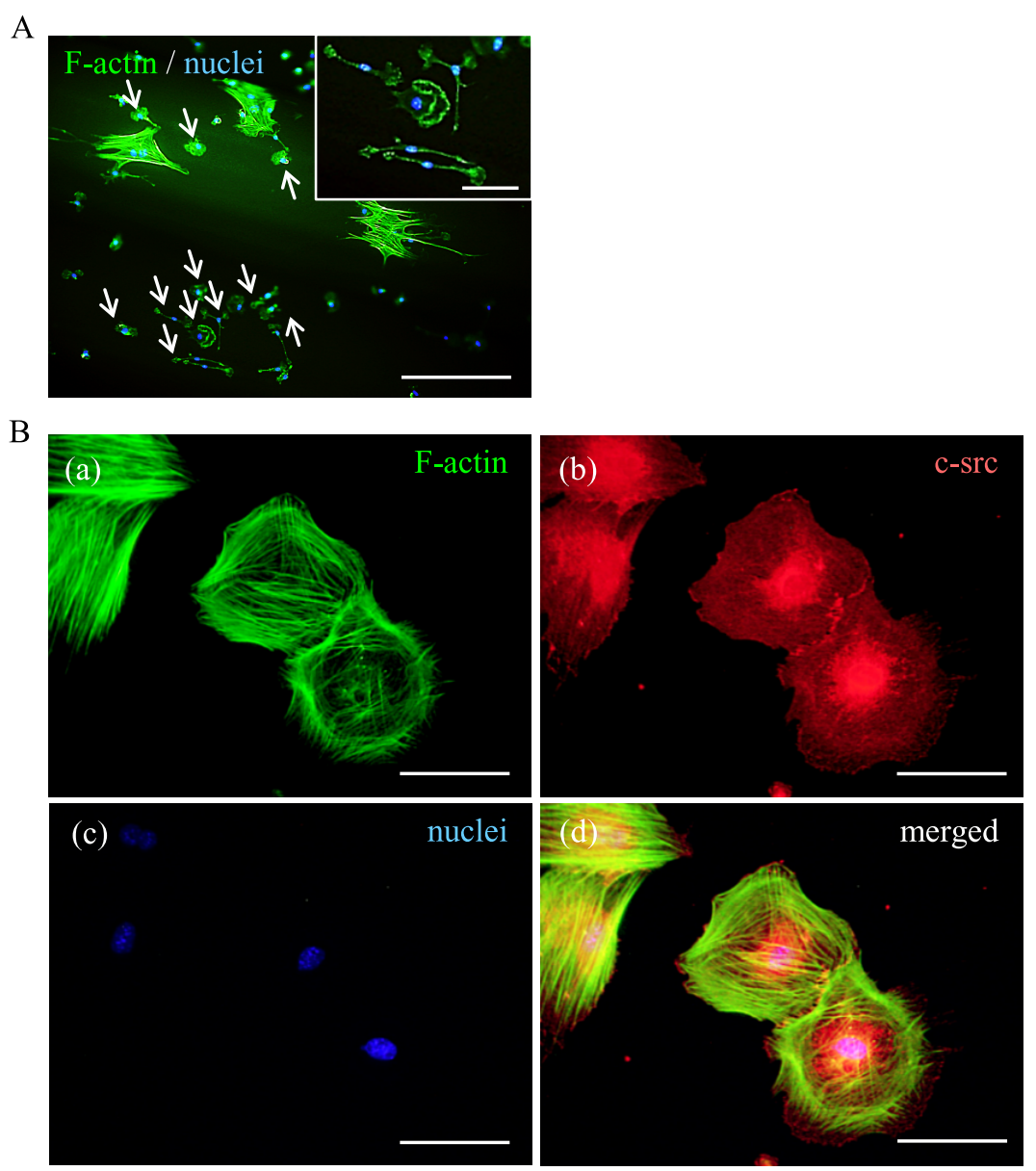

Fig. 2 Fluorescent images of osteoblasts from adult mice. Arrows indicate the contaminated other cell types such as lymphoid cells or myeloid cells. Scale bars $=200 \mu \mathrm{m}$. The inset shows the magnified image of the contaminated cells. Scale bars $=50 \mu \mathrm{m}$. B: Fluorescent images of osteoblasts from juvenile mice stained by (a) F-actin, (b) c-src, (c) nuclei and (d) merged. Scale bars $=100 \mu \mathrm{m}$.

erative ability compared to those of mature osteoblasts from adult mice. ${ }^{16)}$ In other words, adult osteoblasts show poor proliferative activity but high mineralization ability. In this study, juvenile mice (2-week-old) were selected as osteoblast donors to establish superior functions such as high activity levels during proliferation and maturation. Osteoblasts were isolated from calvariae because they are cleaner from the surrounding tissue than long bones, and osteoblasts from calvariae have higher proliferative capacity than those from long bones, and form well-defined mineralized nodules. ${ }^{16)} \mathrm{Be}-$ cause of the contamination of adult osteoblasts with other cell types as mentioned above (Fig. 2A), the subsequent experiments involving the gene expression analysis of osteoblastic differentiation markers and the cell alignment on the oriented collagen substrate were performed for neonatal and juvenile osteoblasts.

\subsection{Osteoblastic maturation of juvenile osteoblasts}

Gene expression analysis for osteoblast-related genes was performed to confirm the maturation of juvenile osteoblasts. Genes encoding bone extracellular matrix proteins are sequentially expressed in the osteoblasts according to the phase of osteoblastic maturation. In particular, COL-I is expressed in the early phase of osteoblastic maturation. $A L P$ and $O S X$ are expressed in the medium phase and BGLAP in the late phase of maturation. ${ }^{17)}$ The expression of $A L P, O S X$, and
$B G L A P$ was significantly high in juvenile osteoblasts than in neonatal osteoblasts (Fig. 3B-D), whereas COL-I expression was low (Fig. 3A), suggesting that juvenile osteoblasts are more mature than neonatal osteoblasts. In particular, BGLAP expression increased drastically in juvenile osteoblasts (approximately 70 times higher compared to that in neonatal osteoblasts). Since mature osteoblasts expressing BGLAP enhanced mineral deposition in vitro, ${ }^{7)}$ juvenile osteoblasts are mature and may have high potential for mineral deposition.

\subsection{Directional control of juvenile osteoblasts for con- struction of anisotropic bone microstructures}

The highly anisotropic microstructure, which is composed of coordinated BAp/collagen composites, is important for anisotropic mechanical function of bone tissue. Since cell orientation is a crucial factor for construction of the anisotropic bone architecture, ${ }^{5,18,19)}$ directional control of osteoblast was driven by oriented collagen substrates. In contrast to the rounded shape of osteoblasts observed on the cover glass (Fig. 2B), osteoblasts plated on oriented collagen substrates showed preferential alignment along the axis of collagen arrangement (Fig. 4A). This suggests that juvenile osteoblasts altered their morphology according to the molecular arrangement of collagen substrates via cell-extracellular matrix interactions mediated by integrins; ${ }^{20)}$ integrins are transmembrane receptors, and signaling of which regulates cytoskeletal orga- 
A

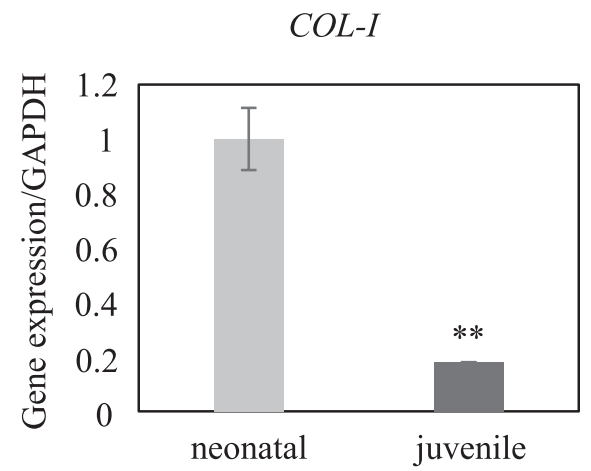

C

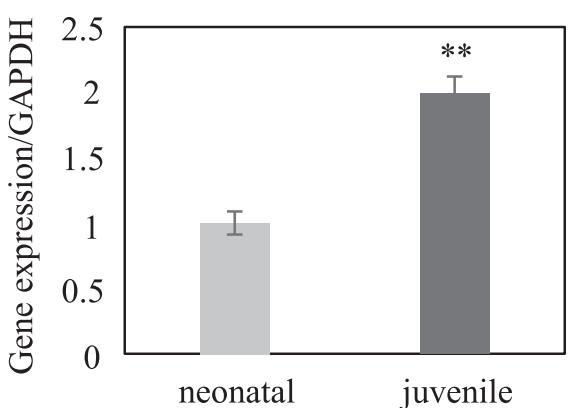

B

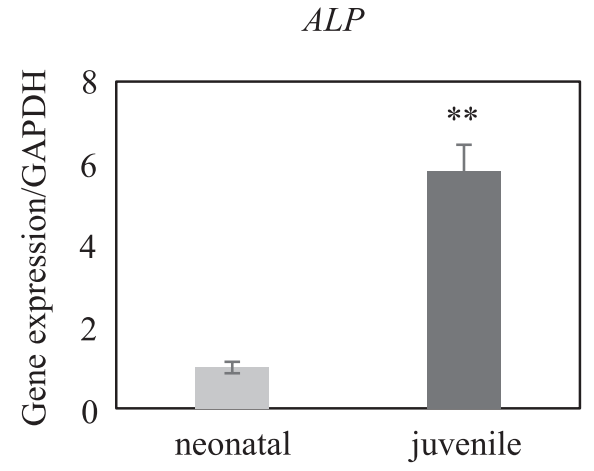

D

BGLAP

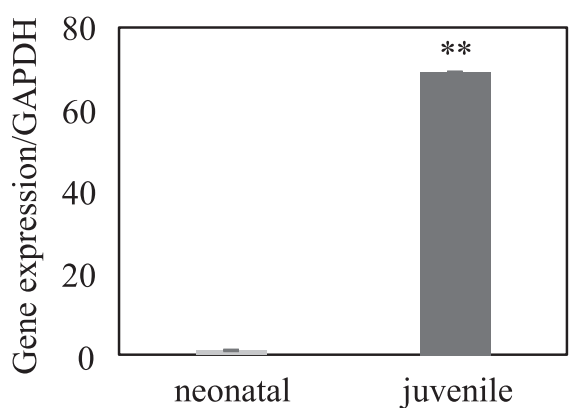

Fig. 3 Quantitative gene expression analysis of osteoblast differentiation markers for osteoblasts from neonatal and juvenile mice. (A) $C O L-I$ (collagen type I), (B) ALP (alkaline phosphatase), (C) OSX (osterix) and (D) BGLAP (osteocalcin). **:p $<0.01$

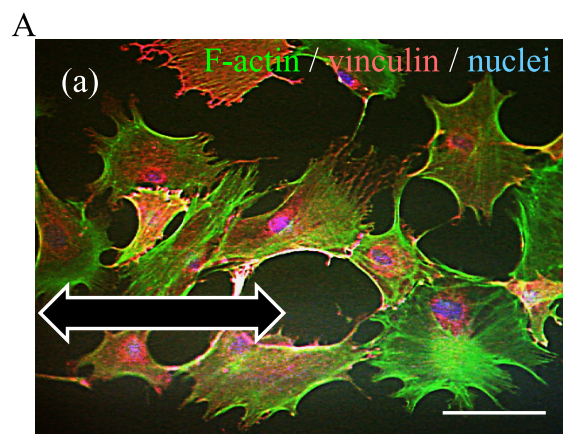

B (a)

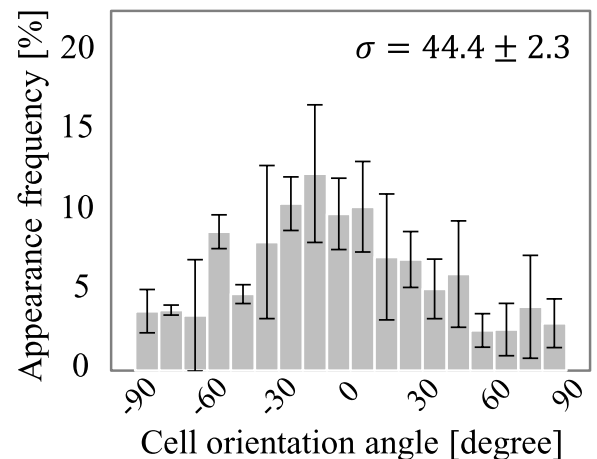

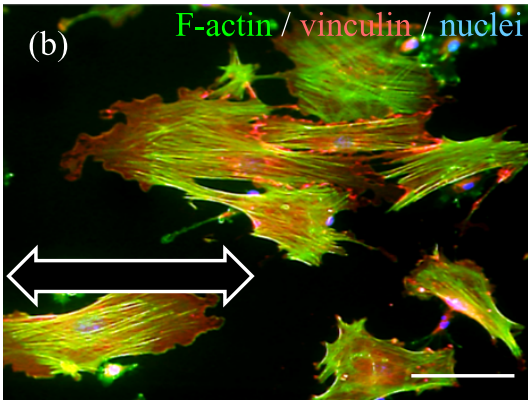

(b)

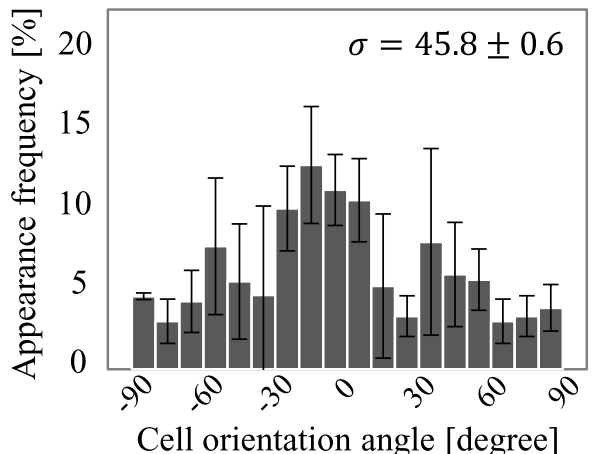

Fig. 4 Osteoblasts from (a) neonatal mice and (b) juvenile mice preferentially aligned along the substrate collagen orientation. Green: F-actin, red: vinculin, blue: nuclei. Arrows indicate the direction of oriented substrate collagen. Scale bars $=100 \mu \mathrm{m}$. B: The angular distribution of (a) neonatal and (b) juvenile osteoblasts. The angular standard deviation $(\sigma)$ representing the degree of cell alignment shows no significant difference between neonatal and juvenile osteoblasts.

nization by mediating extracellular and intracellular communication. Here, juvenile osteoblasts $(\sigma=45.8 \pm 0.6)$ exhibited the same level of cell orientation as neonatal osteoblasts $(\sigma=$
$44.4 \pm 2.3$ ) (Fig. 4B), suggesting that juvenile osteoblasts were capable of constructing the anisotropic bone matrix with the same level as neonatal osteoblasts. These results show 
that mature osteoblasts isolated from juvenile mice could be used for reconstructing the anisotropic bone microstructure in tissue engineering as an ordered microstructure is essential for the mechanical functions of bone tissue.

\section{Conclusions}

Isolation of functional osteoblasts and regulation of their arrangement are crucial for achieving anisotropic bone microstructure, especially for bone regeneration. In this study, an optimal method for construction of the anisotropic bone microstructure by isolation and directional control of mature osteoblasts was established. The major conclusions of this study are as follows.

(1) A homogeneous osteoblast population was successfully obtained from the calvariae of juvenile mice (2-week-old).

(2) Osteoblasts from juvenile mice were significantly mature compared to the neonatal osteoblasts.

(3) The alignment of juvenile osteoblasts was successfully controlled by a bone-mimetic oriented collagen substrate.

\section{Acknowledgements}

This work was supported by the Grants-in-Aid for Scientific Research (S) (JP25220912) from the Japan Society for the Promotion of Science (JSPS) and by the Council for Science, Technology, and Innovation (CSTI), Cross-Ministerial Strategic Innovation Promotion Program (SIP), and Innovative Design/Manufacturing Technologies (Establishment and Validation of the Base for 3D Design \& Additive Manufacturing Standing on the Concepts of "Anisotropy" \& "Customization”) from the New Energy and Industrial Technology Development Organization (NEDO). TN would like to thank the Iketani Science \& Technology Foundation (0281008-A) for partially supporting this research.

\section{REFERENCES}

1) S. Weiner and H.D. Wagner: Annu. Rev. Mater. Sci. 28 (1998) 271298.

2) J. Seto, H.S. Gupta, P. Zaslansky, H.D. Wagner and P. Fratzl: Adv. Funct. Mater. 18 (2008) 1905-1911.

3) T. Ishimoto, T. Nakano, Y. Umakoshi, M. Yamamoto and Y. Tabata: J. Bone Miner. Res. 28 (2013) 1170-1179.

4) T. Nakano, K. Kaibara, T. Ishimoto, Y. Tabata and Y. Umakoshi: Bone 51 (2012) 741-747.

5) A. Matsugaki, Y. Isobe, T. Saku and T. Nakano: J. Biomed. Mater. Res. Part A 103 (2015) 489-499.

6) G. Wong and D.V. Cohn: Nature 252 (1974) 713-715.

7) D. Yang, G.J. Atkins, A.G. Turner, P.H. Anderson and H.A. Morris: J. Steroid Biochem. Mol. Biol. 136 (2013) 166-170.

8) Y. Isobe, T. Kosaka, G. Kuwahara, H. Mikami, T. Saku and S. Kodama: Materials (Basel) 5 (2012) 501-511.

9) A.D. Bakker and J.K. Nulend: Bone Research Protocols: Methods Mol. Biol. 816 (2012) 19-29.

10) C.A. Bashur, L.A. Dahlgren and A.S. Goldstein: Biomaterials 27 (2006) 5681-5688.

11) S. Fujita, M. Ohshima and H. Iwata: J. R. Soc. Interface 6 (2009) S269277.

12) N. I. Fisher: New York Cambridge University Press (1993).

13) J.A. Yagiela and D.M. Woodbury: Anat. Rec. 188 (1977) 287-305.

14) S.M. Thomas and J.S. Brugge: Annu. Rev. Cell Dev. Biol. 13 (1997) 513-609.

15) L. Blanchoin, R.B. Paterski, C. Sykes and J. Plastino: Physiol. Rev. 94 (2014) 235-263.

16) H. Declercq, N.V.D. Vreken, E.D. Maeyer, R. Verbeeck, E. Schacht, L.D. Ridder and M. Cornelissen: Biomaterials 25 (2004) 757-768.

17) J.E. Aubin: Rev. Endocr. Metab. Disord. 2 (2001) 81-94.

18) A. Matsugaki, G. Aramoto, T. Ninomiya, H. Sawada, S. Hata and T. Nakano: Biomaterials 37 (2015) 134-143.

19) A. Matsugaki, N. Fujiwara and T. Nakano: Acta Biomater. 9 (2013) 7227-7235.

20) D. Docheva, C. Popov, P. Alberton and A. Aszodi: Birth Defects Res. C Embryo Today 102 (2014) 13-36. 